\title{
MRI Findings in Children with Headache Seen in a Third-Level Centre
}

\author{
Matteo Chiappedi ${ }^{1}$, Anna Pichiecchio ${ }^{2,3}$, Martina Maria Mensi ${ }^{1}$, and Umberto Balottin ${ }^{1,3}$ \\ ${ }^{1}$ Child Neuropsychiatry Unit, IRCCS Mondino Foundation, Pavia, Italy \\ ${ }^{2}$ Paediatric Neuroradiology Unit, IRCCS Mondino Foundation, Pavia, Italy \\ ${ }^{3}$ University of Pavia, Pavia, Italy
}

The recent review by Jang et al. ${ }^{1}$ confirms a low prevalence of significant intracranial abnormalities found in adult patients with headache, as documented in existing literature.

In our third-level Child Neurology and Psychiatry Unit we do not have an Emergency Department, but patients with headache are quite often seen as outpatients sent by their general paediatrician with a priority access ("deferred urgency"). When deemed necessary after first clinical assessment by the child neuropsychiatrist, these children can be hospitalized to undergo a full evaluation.

In 2017, eighty-four patients (mean age 11 years old) were hospitalized for this reason. They received an in-depth physical examination for associated neurological and psychiatric problems and a detailed history was taken to assess headache frequency; they also underwent different instrumental exams (chosen according to clinical data) and all of them underwent brain-MRI to clarify their diagnosis.

The patients' clinical diagnosis (according to the ICHD III) were: tension-type headache $46 \%$, migraine with aura $17 \%$ and without aura $21 \%$, headache with mixed characteristics $12 \%$, cluster headache $4 \%$. Comorbidities were present in $37.5 \%$ of patients: psychiatric $44 \%$, both psychiatric and neurological $34 \%$, general medicine comorbidities $22 \%$. Brain MRI showed normal findings in $70.3 \%$ of patients, anatomical variants in $8.3 \%$, findings of uncertain pathological significance in $19 \%$ and pathological alterations in about $2.4 \%$ ( 1 case of pseudotumor and 1 of cerebellar astrocytoma correlated with headache). Ear, Nose, Throat (ENT) disorders were documented in $24 \%$ of

Received: July 9, 2019 Accepted: July 25, 2019

$\triangle$ Correspondence: Matteo Chiappedi, MD, PhD

Child Neuropsychiatry Unit, National Neurological Institute IRCCS C. Mondino Foundation, Via Mondino 2, 27100 Pavia PV, Italy

Tel: +390382 380285, E-mail: matteo.chiappedi@mondino.it

(a) This is an Open Access article distributed under the terms of the Creative Commons Attribution Non-Commercial License (https://creativecommons.org/licenses/bync/4.0) which permits unrestricted non-commercial use, distribution, and reproduction in any medium, provided the original work is properly cited. patients: 5\% nasopharynx hypertrophy, 19\% paranasal sinus disease, $5 \%$ mastoid involvement.

These data are interesting for two main reasons. First, it is important to highlight that $24 \%$ had ENT disorders not detected during clinical assessment; it is worth noting that these unrecognized ENT disorders were easily treatable (at follow up, this treatment also produced a cessation of headache). This means that for these patients MRI was in fact an exam that changed the treatment prescribed and/or increased it effectiveness. Second, the prevalence of significant alterations in our study was lower than the usually reported. ${ }^{2}$

Jang et al. ${ }^{1}$ describe the existing concerns about performing unnecessary neuroradiological exams. This poses serious ethical concerns, given on one hand the importance of the early identification of potentially life-threatening diseases and on the other hand the cost of these procedures both for the child (procedural sedation, X-rays in the case of CT) and for the health system. Moreover, inconsequential findings can lead to further unnecessary examinations and can be used as a defence to avoid the exploration of other potentially relevant factors, such as the psychological functioning of the child. ${ }^{3,4}$ This is especially important because of the relevance of psychological factors for treatment and prognosis of childhood headache is well established. ${ }^{5}$

Our data support the need of robust prospective studies to better define clinical factors predicting (or excluding) the utility of MRI in children and adolescents with headache.

\section{Conflicts of Interest}

The authors have no potential conflicts of interest to disclose.

\section{ORCID iD}

Matteo Chiappedi https://orcid.org/0000-0002-2682-5006

\section{REFERENCES}

1. Jang YE, Cho EY, Choi HY, Kim SM, Park HY. Diagnostic neuroimag- 
ing in headache patients: a systematic review and meta-analysis. Psychiatry Investig 2019;16:407-417.

2. Conicella E, Raucci U, Vanacore N. The child with headache in a pediatric emergency department. Headache 2008;48:1005-1011.

3. Balottin L, Mannarini S, Candeloro D, Mita A, Chiappedi M, Balottin $\mathrm{U}$. Rorschach evaluation of personality and emotional characteristics in adolescents with migraine versus epilepsy and controls. Front Neu- rol 2018;9:160.

4. Balottin U, Chiappedi M, Rossi M, Termine C, Nappi G. Childhood and adolescent migraine: a neuropsychiatric disorder? Med Hypotheses 2011;76:778-781.

5. Chiappedi M, Mensi MM, Termine C, Balottin U. Psychological therapy in adolescents with chronic daily headache. Current Pain Headache Rep 2016;20:3. 